\title{
Drug Susceptibility of 33 Reference Strains of Slowly Growing Mycobacteria to 19 Antimicrobial Agents
}

\author{
Hui Pang, ${ }^{1}$ Yi Jiang, ${ }^{2,3}$ and Kanglin Wan ${ }^{2,3}$ \\ ${ }^{1}$ Department of Immunology, Changzhi Medical College, Changzhi, Shanxi 046000, China \\ ${ }^{2}$ State Key Laboratory for Infectious Disease Prevention and Control, National Institute for \\ Communicable Disease Control and Prevention, Chinese Center for Disease Control and Prevention, Beijing 102206, China \\ ${ }^{3}$ Collaborative Innovation Center for Diagnosis and Treatment of Infectious Diseases, Hangzhou, Zhejiang 310000, China
}

Correspondence should be addressed to Kanglin Wan; wankanglin@icdc.cn

Received 23 December 2016; Revised 24 March 2017; Accepted 6 April 2017; Published 20 April 2017

Academic Editor: Frederick D. Quinn

Copyright (C) 2017 Hui Pang et al. This is an open access article distributed under the Creative Commons Attribution License, which permits unrestricted use, distribution, and reproduction in any medium, provided the original work is properly cited.

Objectives. Slowly growing mycobacteria (SGM) are prevalent worldwide and cause an extensive spectrum of diseases. Methods. In this study, the antimicrobial susceptibility of 33 reference strains of SGM to 19 antimicrobial agents was tested using a modified microdilution method. Results. Cefmetazole (32/33) and azithromycin (32/33) exhibited the highest antimicrobial activity, and dapsone (9/33) exhibited the lowest activity against the tested strains. Cefoxitin (30/33), cefoperazone (28/33), and cefepime (28/33) were effective against a high proportion of strains, and macrolides were also highly effective as well as offering the benefit of convenient oral administration to patients. Linezolid (27/33), meropenem (26/33), sulfamethoxazole (26/33), and tigecycline (25/33) showed the highest activity; clofazimine (20/33) and doxycycline (18/33) showed intermediate activity; and rifapentine (13/33), rifabutin (13/33), and minocycline (11/33) showed low antimicrobial activity, closely followed by thioacetazone (10/33) and pasiniazid (10/33), against the tested organisms. According to their susceptibility profiles, the slowly growing species Mycobacterium avium and Mycobacterium simiae were the least susceptible to the tested drugs, whereas Mycobacterium intracellulare, Mycobacterium asiaticum, Mycobacterium scrofulaceum, Mycobacterium szulgai, Mycobacterium branderi, and Mycobacterium holsaticum were the most susceptible. Conclusions. In summary, cephalosporins and macrolides, particularly cefmetazole, azithromycin, clarithromycin, and roxithromycin, showed good antimicrobial activity against the reference strains of SGM.

\section{Introduction}

Slowly growing mycobacteria (SGM) species are ubiquitous organisms that are widely distributed in the environment [1], not only in tap water, soil, dust, and food products but also in domestic and wild animals [2]. SGM form colonies visible to the naked eye in more than 7 days on subculture media [3]. SGM comprise some common species, such as the Mycobacterium avium complex (Mycobacterium avium, Mycobacterium intracellulare, and Mycobacterium chimaera), Mycobacterium kansasii, Mycobacterium haemophilum, Mycobacterium marinum, and Mycobacterium ulcerans, in addition to some less common pathogens, such as Mycobacterium scrofulaceum, Mycobacterium simiae, Mycobacterium malmoense and Mycobacterium xenopi. Mycobacterium xenopi is largely distributed in Canada and northern Europe [4]. Slowly growing species were the first nontuberculous mycobacteria (NTM) to be recognized as causing chronic lung disease $[4,5]$, which may bring about diverse infections from minor sicknesses to serious widespread disorders [6].

At present, standard therapeutic strategies to treat SGM infections are lacking. In this study, 19 new antimicrobial agents were tested against 33 reference SGM pathogens using a modified broth microdilution method with the aim of identifying optimal schemes according to the Clinical Laboratory Standards Institute (CLSI) (USA) [7, 8] and World Health Organization (WHO) [9] guidelines.

\section{Materials and Methods}

2.1. Reference Strains. Thirty-three international reference SGM strains were purchased from Deutsche Sammlung von 
Mikroorganismen und Zellkulturen (DSMZ) and the American Type Culture Collection (ATCC), including Mycobacterium avium, Mycobacterium intracellulare, Mycobacterium shimoidei, Mycobacterium farcinogenes, and Mycobacterium simiae (Table 1). These strains were cultured at the appropriate temperatures.

2.2. Antimicrobial Agents. Nineteen chemicals were purchased from Sigma-Aldrich Company: cefoxitin (FOX), cefoperazone (CFP), cefmetazole (CMZ), cefepime (FEP), rifapentine (RPT), rifabutin (RBT), azithromycin (AZM), clarithromycin (CLR), roxithromycin (ROX), thioacetazone (THI), doxycycline (DOX), minocycline (MIN), tigecycline (TIG), meropenem (MEM), clofazimine (CLO), sulfamethoxazole (SMZ), pasiniazid (PASI), linezolid (LNZ), and dapsone (DAP). All of the antituberculous agents were freshly prepared.

2.3. Drug Susceptibility Test. SGM strains were incubated using Difco Middlebrook 7H10 Agar (BD company) with $5 \%$ oleic acid-albumin-dextrose-catalase (OADC) [8]. The drug sensitivity tests were performed using a cation-adjusted Mueller-Hinton (CAMH) broth microdilution method, with the addition of $5 \%$ OADC, according to the CLSI standard operating procedure [8]. All of the experiments were performed in 96-well microplates and repeated. The minimum inhibitory concentration (MIC) for each antibiotic for each strain was the mean of two experiments. Firstly, the bacterial suspensions were prepared as follows: bacterial inocula were adjusted with normal saline to a density of a $0.5 \mathrm{McF}$ arland standard with an inoculum density of approximately $1 \times 10^{7}$ colony forming units $(\mathrm{CFU}) / \mathrm{mL}$; then $50 \mu \mathrm{L}$ of the bacterial suspension was mixed with $10 \mathrm{~mL}$ of CAMH and 5\% OADC broth for a 1:200 dilution. Secondly, $100 \mu \mathrm{L}$ of $\mathrm{CAMH}$ and $5 \%$ OADC medium were added to each well of a 96-well microplate, with the exception of the first well of every row to which $180 \mu \mathrm{L}$ of medium and a $20 \mu \mathrm{L}$ drug dilution were added. The solution in the first well was successively diluted into subsequent wells, up to the 11th well. The 12th well in every row was used as a blank control. Finally, $100 \mu \mathrm{L}$ of the bacterial dilution was added to all of the wells. The ultimate volume in each well was $200 \mu \mathrm{L}$. All of the 96-well microplates were sealed in a plastic bag and incubated at $37^{\circ} \mathrm{C}$. The concentrations of sulfamethoxazole, dapsone, cefoxitin, cefmetazole, cefoperazone, cefepime, thioacetazone, pasiniazid, minocycline, doxycycline, tigecycline, and meropenem were $0.25-256 \mu \mathrm{g} / \mathrm{mL}$; the concentrations of clarithromycin, azithromycin, roxithromycin, clofazimine, rifapentine, and rifabutin were $0.03-32 \mu \mathrm{g} / \mathrm{mL}$; and the concentration of linezolid was $0.06-64 \mu \mathrm{g} / \mathrm{mL}$. Two negative controls were applied: a no drug control (CAMH + OADC + bacteria) and a no bacteria control (barely CAMH and OADC) [10]. The MIC breakpoints of the drugs exhibiting susceptibility, moderate susceptibility, and resistance were assigned according to the CLSI [7, 8] and WHO [9] guidelines (Table 2).

\section{Results}

The antimicrobial susceptibility profiles of the 33 SGM reference species to 19 antibacterial agents are presented in Table 1. Cephalosporins including cefoxitin (30/33, 90.91\%), cefoperazone $(28 / 33,84.85 \%)$, cefmetazole $(32 / 33,96.97 \%)$, and cefepime $(28 / 33,84.85 \%)$ exhibited high activity against the tested strains. Macrolide antibiotics including azithromycin (32/33, 96.97\%), clarithromycin (30/33, 90.91\%), and roxithromycin $(31 / 33,93.94 \%)$ were also effective against the SGM strains. Linezolid (27/33, 81.82\%), meropenem (26/33, $78.79 \%)$, and sulfamethoxazole $(26 / 33,78.79 \%)$ showed similar levels of activity against the tested strains, and clofazimine $(20 / 33,60.61 \%)$ inhibited most of the SGM strains. The tetracyclines, doxycycline $(18 / 33,54.55 \%)$, minocycline $(11 / 33$, $33.33 \%)$, and tigecycline $(25 / 33,75.76 \%)$, exhibited different levels of activity against the SGM standard species, whereas rifapentine $(13 / 33,39.39 \%)$ and rifabutin (13/33, 39.39\%) showed weak antimicrobial activity against the SGM, as did thioacetazone $(10 / 33,30.30 \%)$, pasiniazid (10/33, 30.30\%), and dapsone $(9 / 33,27.27 \%)$.

The drug susceptibility profiles of the tested organisms revealed that Mycobacterium avium and Mycobacterium simiae were the least susceptible to the tested drugs, whereas Mycobacterium intracellulare, Mycobacterium asiaticum, Mycobacterium scrofulaceum, Mycobacterium szulgai, Mycobacterium branderi, and Mycobacterium holsaticum were the most susceptible (Table 3 and Figure 1). Among the Mycobacterium avium complex, Mycobacterium avium was the most resistant to the tested drugs, whereas Mycobacterium intracellulare was the most susceptible (Figure 2). Azithromycin was identified as the most effective antimicrobial agent against SGM species among the drugs tested, and dapsone was the least effective.

\section{Discussion}

In this study, 19 antimicrobial susceptibility tests were performed against 33 SGM organisms by a Microplate Alamar Blue Assay. The current first-line drugs for the treatment of nontuberculous mycobacteria are capreomycin, clarithromycin, and rifampin. And the current second-line drugs for the treatment of nontuberculous mycobacteria are moxifloxacin, linezolid, amikacin, ciprofloxacin, ethambutol, isoniazid, rifabutin, streptomycin, and trimethoprim-sulfamethoxazole [7]. Our findings indicated that cephalosporins and macrolides, particularly cefmetazole, azithromycin, clarithromycin, and roxithromycin, showed effective antimicrobial activity against the tested strains.

In recent studies $[4,11-15]$, cefoxitin and meropenem have been reported to show some activity against Mycobacterium abscessus, Mycobacterium chelonae, and Mycobacterium fortuitum, whereas Mycobacterium kansasii has been shown to be susceptible to clarithromycin and linezolid. Macrolides were active against isolates of Mycobacterium avium $[12,16,17]$, and tigecycline has been demonstrated to exhibit high level antimicrobial activity against RGM in vitro [18]. In other studies, Mycobacterium kansasii was reported to 


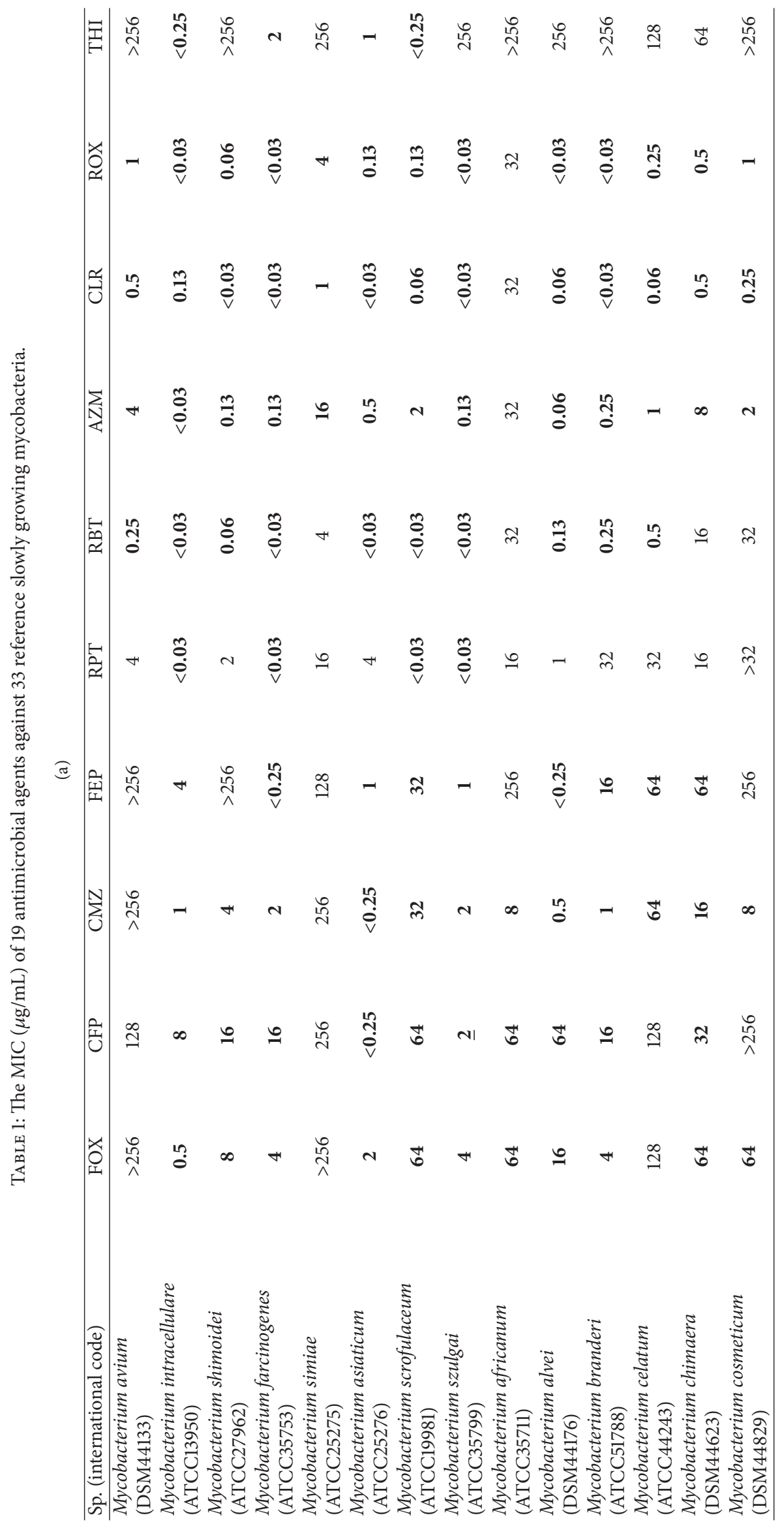




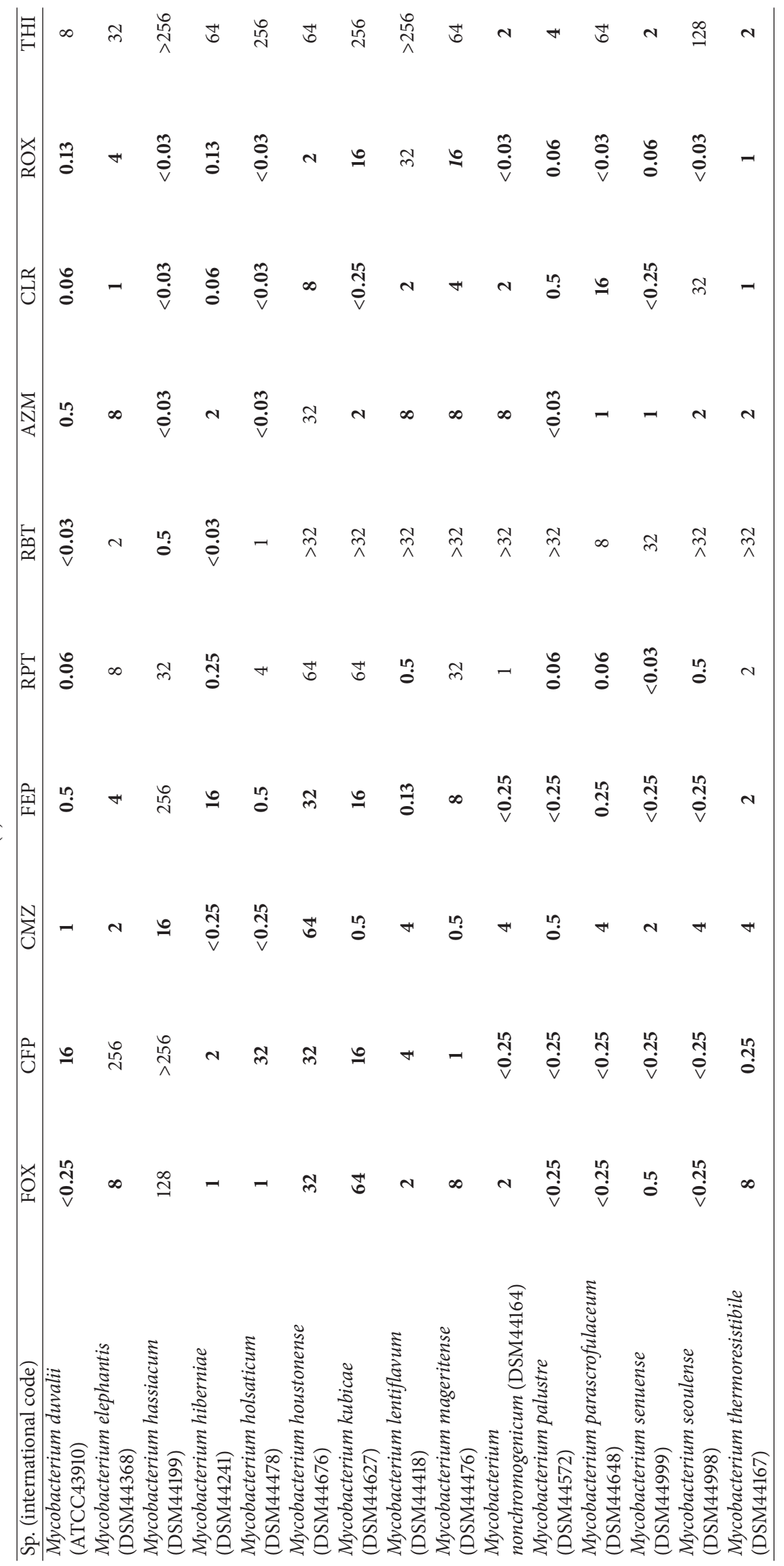




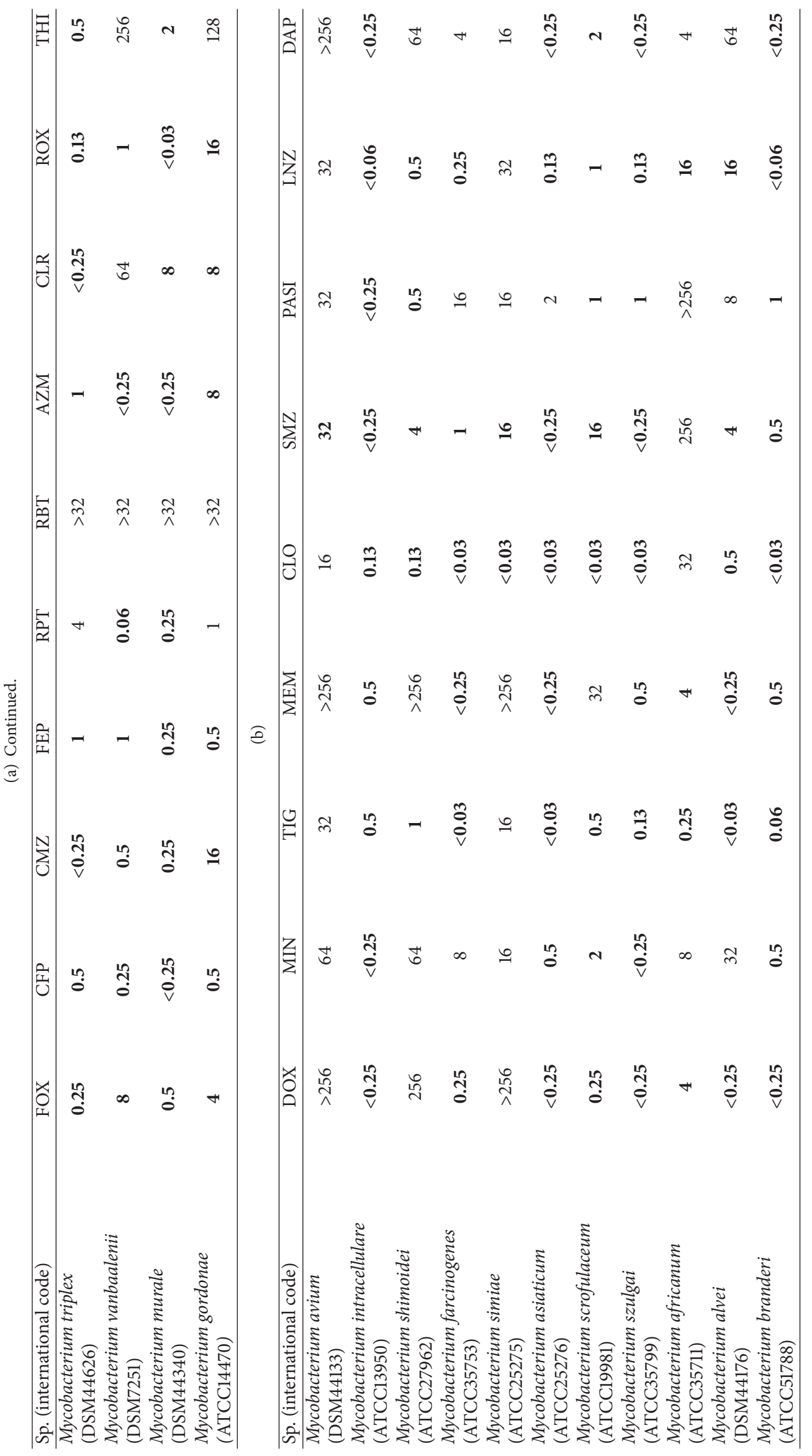




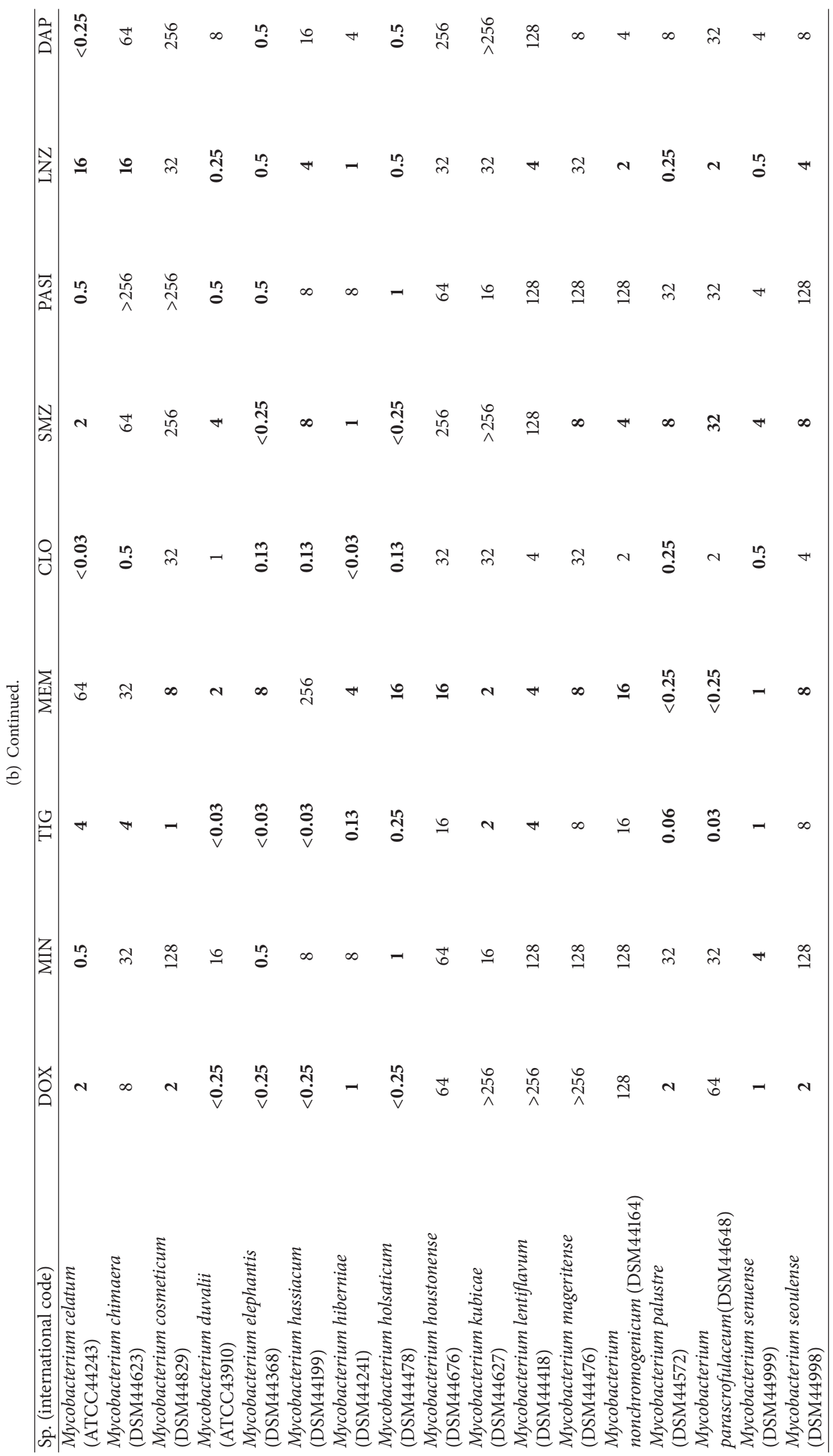




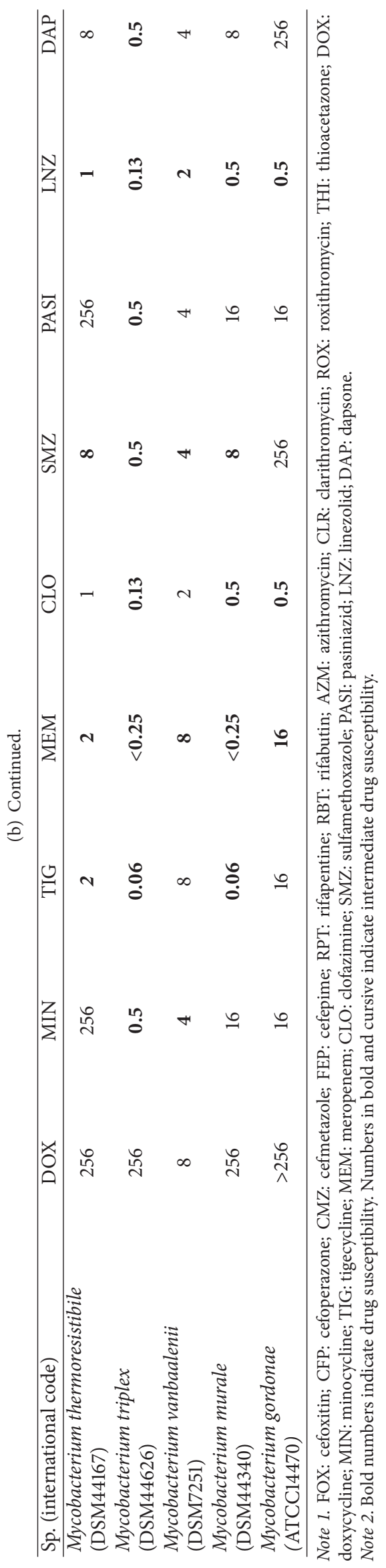


TABLE 2: The MIC ( $\mu \mathrm{g} / \mathrm{mL})$ breakpoints of 19 antibacterial agents.

\begin{tabular}{|c|c|c|c|}
\hline & Susceptibility & Intermediate susceptibility & Resistance \\
\hline Cefoxitin & $\leq 16$ & $32-64$ & $\geq 128$ \\
\hline Cefoperazone & $\leq 16$ & $32-64$ & $\geq 128$ \\
\hline Cefmetazole & $\leq 16$ & $32-64$ & $\geq 128$ \\
\hline Cefepime & $\leq 16$ & $32-64$ & $\geq 128$ \\
\hline Rifapentine & - & - & $>1$ \\
\hline Rifabutin & - & - & $>2$ \\
\hline Azithromycin & $\leq 8$ & 16 & $\geq 32$ \\
\hline Clarithromycin & $\leq 8$ & 16 & $\geq 32$ \\
\hline Roxithromycin & $\leq 8$ & 16 & $\geq 32$ \\
\hline Thioacetazone & - & - & $\geq 8$ \\
\hline Doxycycline & $\leq 1$ & $2-4$ & $\geq 8$ \\
\hline Meropenem & $\leq 4$ & $8-16$ & $\geq 32$ \\
\hline Clofazimine & - & - & $\geq 1$ \\
\hline Sulfamethoxazole & $\leq 38$ & - & $\geq 76$ \\
\hline Pasiniazid & - & - & $\geq 2$ \\
\hline Minocycline & $\leq 1$ & $2-4$ & $\geq 8$ \\
\hline Linezolid & $\leq 8$ & 16 & $\geq 32$ \\
\hline Dapsone & - & - & $\geq 4$ \\
\hline Tigecycline & $\leq 1$ & $2-4$ & $\geq 8$ \\
\hline
\end{tabular}

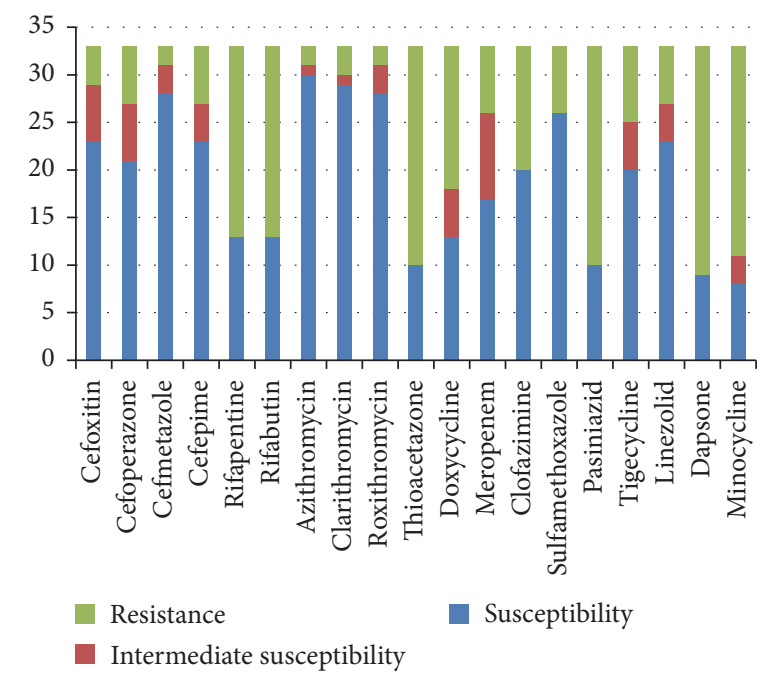

FIGURE 1: The sensitivity profiles of 33 reference slowly growing mycobacteria to 19 antimicrobial agents.

be the most susceptible NTM species in vitro [19], and Mycobacterium simiae was found to be resistant to clarithromycin, doxycycline, and sulfamethoxazole [20, 21]. However, few studies have tested the activity of cefoperazone, cefmetazole, and cefepime against SGM. In our study, cephalosporins were found to be effective antimicrobial agents and cefmetazole in particular was identified as a good candidate for the treatment of SGM infections. In previous research $[15,22]$, clarithromycin has been widely used as an antimicrobial agent to SGM, whereas azithromycin and roxithromycin have rarely been tested. Among the tetracyclines, tigecycline was found to be the most effective against SGM. Previous studies have reported that Mycobacterium kansasii was 100\% resistant to doxycycline, and Mycobacterium simiae isolates were $100 \%$ resistant to clarithromycin, doxycycline, and sulfamethoxazole.

Mycobacterium avium and Mycobacterium intracellulare are important members of the SGM. Macrolides and sulfamethoxazole are recognized as useful drugs against Mycobacterium avium and Mycobacterium intracellulare, but rifapentine is ineffective against Mycobacterium avium. Mycobacterium chimaera, a recently described species distinct from 


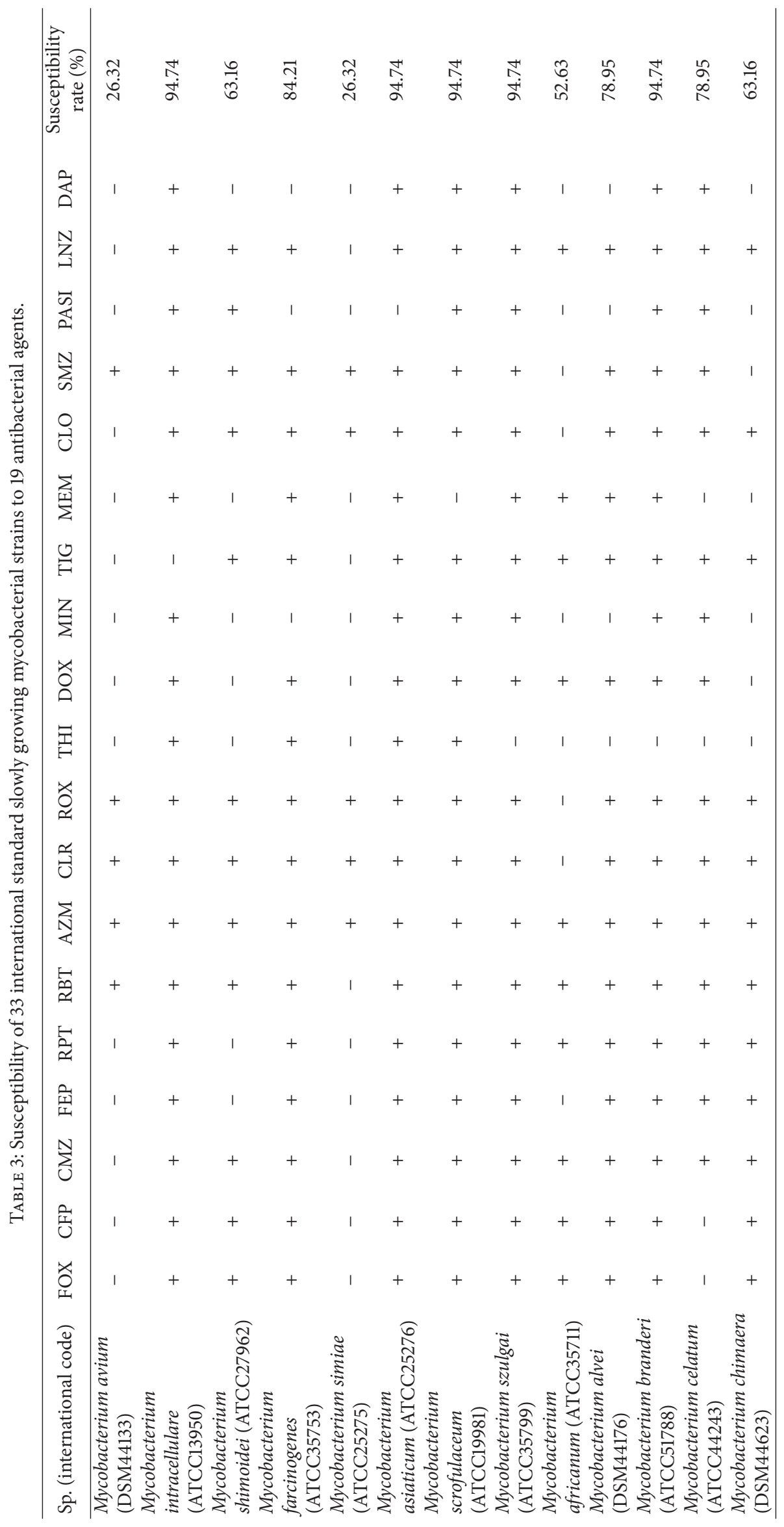




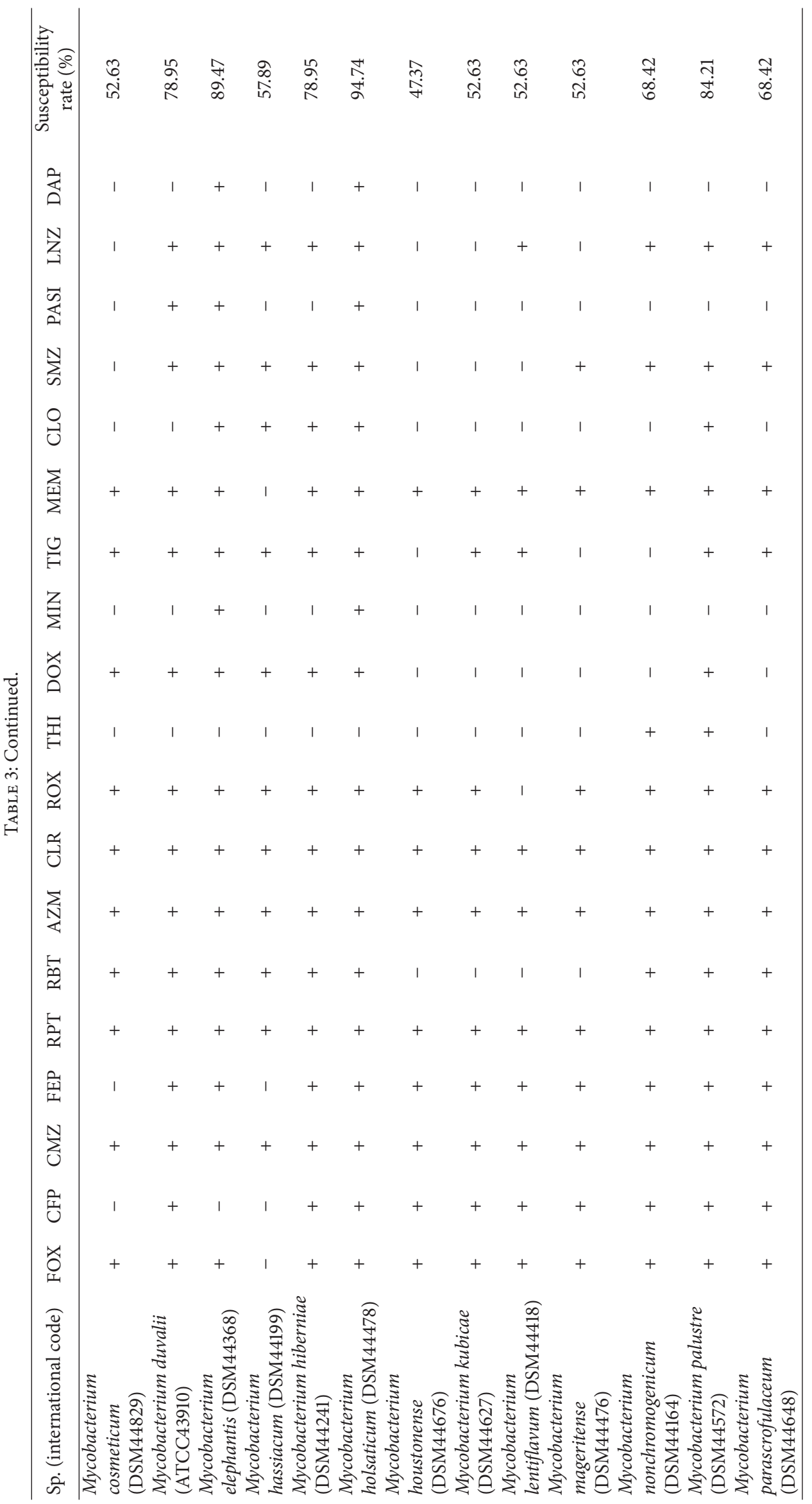




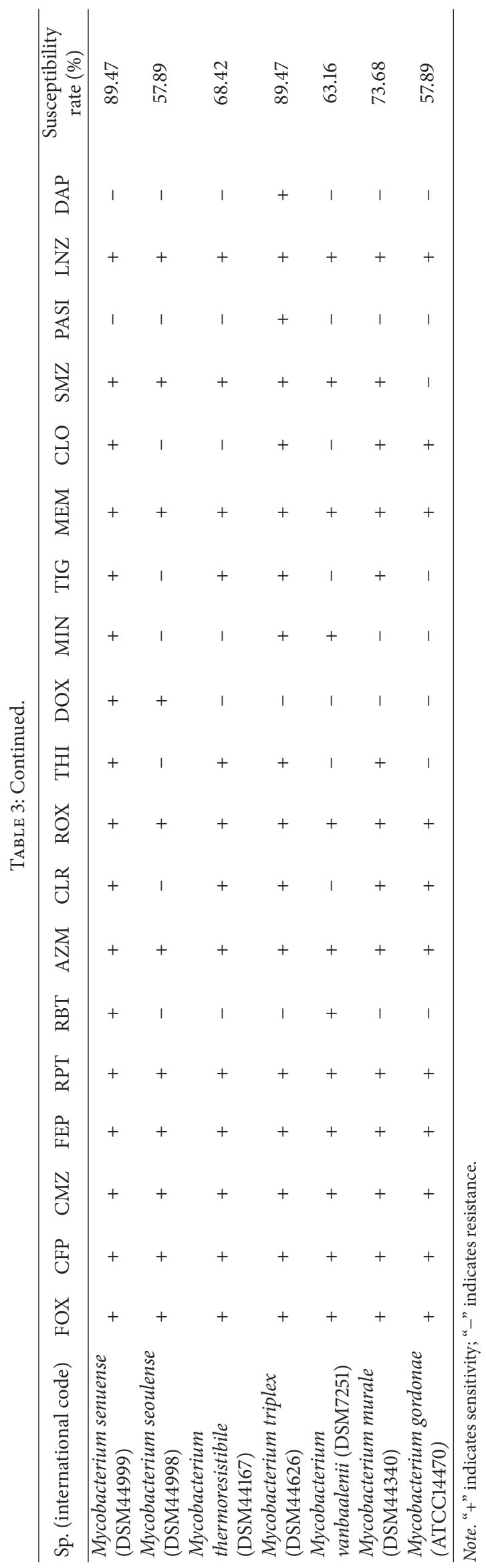




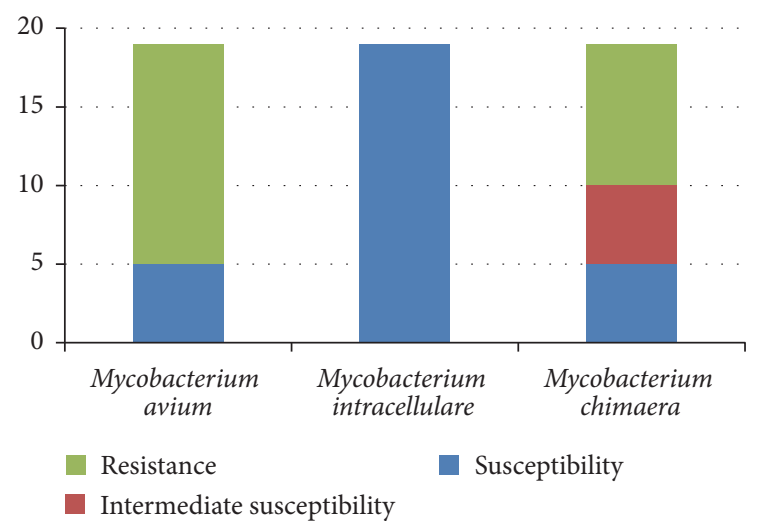

FIGURE 2: The sensitivity profiles of the Mycobacterium avium complex to 19 antimicrobial agents.

Mycobacterium intracellulare, is regarded as less virulent than Mycobacterium intracellulare [23, 24], but neither rifapentine nor rifabutin was effective against Mycobacterium chimaera.

Mycobacterium simiae was highly resistant to the tested drugs. It was first isolated from monkeys in 1965 and is now most frequently isolated from human respiratory specimens $[25,26]$, predominantly being reported in the southwest of the United States and Middle Eastern countries, including Israel and Iran [27].

\section{Conclusions}

Our findings present the drug susceptibility profiles of representative SGM species to a range of antimicrobial agents and provide insight into potentially effective therapeutic strategies. In the future, susceptibility testing of clinical isolates may help to tailor therapeutic strategies to individual patients. Combination therapy should also be explored as a means to increase the efficacy of drug treatment against SGM pathogens. Furthermore, the synergistic activity of some drugs will be analyzed, and drug susceptibility in vivo response must be performed in our recent research.

\section{Conflicts of Interest}

The authors have declared that no conflicts of interest exist.

\section{Acknowledgments}

This paper is financially supported by the Project of the National Key Program of Mega Infectious Diseases (no. 2013ZX10004-101), the Key Project of the State Key Laboratory for Infectious Disease Prevention and Control (no. 2014SKLID104), Science and Technology of Shanxi Province for Youth Science Foundation (no. 2016021161), a Science and Technology Innovation Team support project (no. CX201412) from Changzhi Medical College, and innovation project of university students in Shanxi Province (no. 2016303). The authors thank the staffs of National Institute for Communicable Disease Control and Prevention, Chinese Center for Disease Control and Prevention.

\section{References}

[1] S. Y. Aboagye, E. Danso, K. A. Ampah et al., "Isolation of nontuberculous mycobacteria from the environment of ghanian communities where buruli ulcer is endemic," Applied and Environmental Microbiology, vol. 82, no. 14, pp. 4320-4329, 2016.

[2] D. Azadi, H. Shojaei, M. Pourchangiz, R. Dibaj, M. Davarpanah, and AD. Naser, "Species diversity and molecular characterization of nontuberculous mycobacteria in hospital water system of a developing country, Iran," Microbial Pathogenesis, vol. 100, pp. 62-69, 2016.

[3] T. Tsukatani, H. Suenaga, M. Shiga et al., "Rapid susceptibility testing for slowly growing nontuberculous mycobacteria using a colorimetric microbial viability assay based on the reduction of water-soluble tetrazolium WST-1," European Journal of Clinical Microbiology and Infectious Diseases, vol. 34, no. 10, pp. 19651973, 2015.

[4] J. V. Philley and D. E. Griffith, "Treatment of slowly growing mycobacteria," Clinics in Chest Medicine, vol. 36, no. 1, pp. 7990, 2015.

[5] B. E. Ferro, J. van Ingen, M. Wattenberg, D. van Soolingen, and J. W. Mouton, "Time-kill kinetics of slowly growing mycobacteria common in pulmonary disease," Journal of Antimicrobial Chemotherapy, vol. 70, no. 10, Article ID dkv180, pp. 2838-2843, 2015.

[6] D. E. Griffith, "Nontuberculous mycobacterial lung disease," Current Opinion in Infectious Diseases, vol. 23, no. 2, pp. 185190, 2010

[7] Clinical and Laboratory Standards Institute, Susceptibility Testing of Mycobacteria, Nocardiae, and Other Aerobic Actinomycetes; Approved Standard-Second Edition, CLSI Document, M24A2, 2011.

[8] Clinical and Laboratory Standards Institute, Performance standards for Antimicrobial Susceptibility Testing; Twenty First Informational Supplement, CLSI Document M100-S25, Clinical and Laboratory Standards Institute, 2015.

[9] World Health Organization, Policy Guidance on Drug-Susceptibility Testing (DST) of Second-Line Antituberculosis Drugs, WHO Document, 2008.

[10] S. Foongladda, S. Pholwat, B. Eampokalap, P. Kiratisin, and R. Sutthent, "Multi-probe real-time PCR identification of common mycobacterium species in blood culture broth," Journal of Molecular Diagnostics, vol. 11, no. 1, pp. 42-48, 2009.

[11] H. Pang, G. Li, X. Zhao, H. Liu, K. Wan, and P. Yu, "Drug susceptibility testing of 31 antimicrobial agents on rapidly growing mycobacteria isolates from China," BioMed Research International, vol. 2015, Article ID 419392, 2015.

[12] P. Heidarieh, M. Mirsaeidi, M. Hashemzadeh et al., "In Vitro Antimicrobial Susceptibility of Nontuberculous Mycobacteria in Iran," Microbial Drug Resistance, vol. 22, no. 2, pp. 172-178, 2016.

[13] H. Pang, G. Li, L. Wan et al., "In vitro drug susceptibility of 40 international reference rapidly growing mycobacteria to 20 antimicrobial agents," International Journal of Clinical and Experimental Medicine, vol. 8, no. 9, pp. 15423-15431, 2015.

[14] M. Jankovic, L. Zmak, V. Krajinovic et al., "A fatal Mycobacterium chelonae infection in an immunosuppressed patient with systemic lupus erythematosus and concomitant Fahr's syndrome," Journal of Infection and Chemotherapy, vol. 17, no. 2, pp. 264-267, 2011.

[15] Z. Gitti, E. Mantadakis, S. Maraki, and G. Samonis, "Clinical significance and antibiotic susceptibilities of nontuberculous 
mycobacteria from patients in Crete, Greece," Future Microbiology, vol. 6, no. 9, pp. 1099-1109, 2011.

[16] M. Shah, N. Relhan, A. E. Kuriyan et al., "Endophthalmitis Caused by Nontuberculous Mycobacterium: Clinical Features, Antimicrobial Susceptibilities, and Treatment Outcomes," American Journal of Ophthalmology, vol. 168, pp. 150-156, 2016.

[17] H. I. Bax, I. A. J. M. Bakker-Woudenberg, M. T. T. Kate, A. Verbon, and J. E. M. De Steenwinkelb, "Tigecycline potentiates clarithromycin activity against mycobacterium avium in vitro," Antimicrobial Agents and Chemotherapy, vol. 60, no. 4, pp. 2577-2579, 2016.

[18] S. S. Tang, D. C. Lye, R. Jureen, L.-H. Sng, and L. Y. Hsu, "Rapidly growing mycobacteria in Singapore, 2006-2011," Clinical Microbiology and Infection, vol. 21, no. 3, pp. 236-241, 2015.

[19] M. Hombach, A. Somoskövi, R. Hömke, C. Ritter, and E. C. Böttger, "Drug susceptibility distributions in slowly growing non-tuberculous mycobacteria using MGIT 960 TB eXiST," International Journal of Medical Microbiology, vol. 303, no. 5, pp. 270-276, 2013.

[20] S. Cowman, K. Burns, S. Benson, R. Wilson, and M. R. Loebinger, "The antimicrobial susceptibility of non-tuberculous mycobacteria," Journal of Infection, vol. 72, no. 3, pp. 324-331, 2016.

[21] P. García-Martos, L. García-Agudo, E. González-Moya, F. Galán, and M. Rodríguez-Iglesias, "Infections due to Mycobacterium simiae," Enfermedades Infecciosas y Microbiologia Clinica, vol. 33, no. 8, pp. e37-e43, 2015.

[22] I. Ahmed, K. Jabeen, and R. Hasan, "Identification of nontuberculous mycobacteria isolated from clinical specimens at a tertiary care hospital: A cross-sectional study," BMC Infectious Diseases, vol. 13, no. 1, article no. 493, 2013.

[23] S. M. Moon, S. Y. Kim, B. W. Jhun et al., "Clinical characteristics and treatment outcomes of pulmonary disease caused by Mycobacterium chimaera," Diagnostic Microbiology and Infectious Disease, vol. 86, no. 4, pp. 382-384, 2016.

[24] R. J. Wallace Jr., E. Iakhiaeva, M. D. Williams et al., "Absence of Mycobacterium intracellulare and presence of Mycobacterium chimaera in household water and biofilm samples of patients in the United States with Mycobacterium avium complex respiratory disease," Journal of Clinical Microbiology, vol. 51, no. 6, pp. 1747-1752, 2013.

[25] S. H. Jeong, S.-Y. Kim, H. Lee et al., "Nontuberculous mycobacterial lung disease caused by mycobacterium simiae: The first reported case in South Korea," Tuberculosis and Respiratory Diseases, vol. 78, no. 4, pp. 432-435, 2015.

[26] D. E. Griffith, T. Aksamit, B. A. Brown-Elliott et al., "An official ATS/IDSA statement: Diagnosis, treatment, and prevention of nontuberculous mycobacterial diseases," American Journal of Respiratory and Critical Care Medicine, vol. 175, no. 4, pp. 367416, 2007.

[27] A. A. Velayati, P. Farnia, M. Mozafari et al., "Molecular epidemiology of nontuberculous mycobacteria isolates from clinical and environmental sources of a metropolitan city," PLoS ONE, vol. 9, no. 12, Article ID el14428, 2014. 


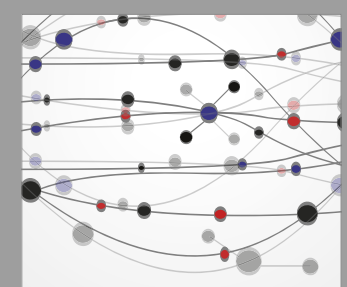

The Scientific World Journal
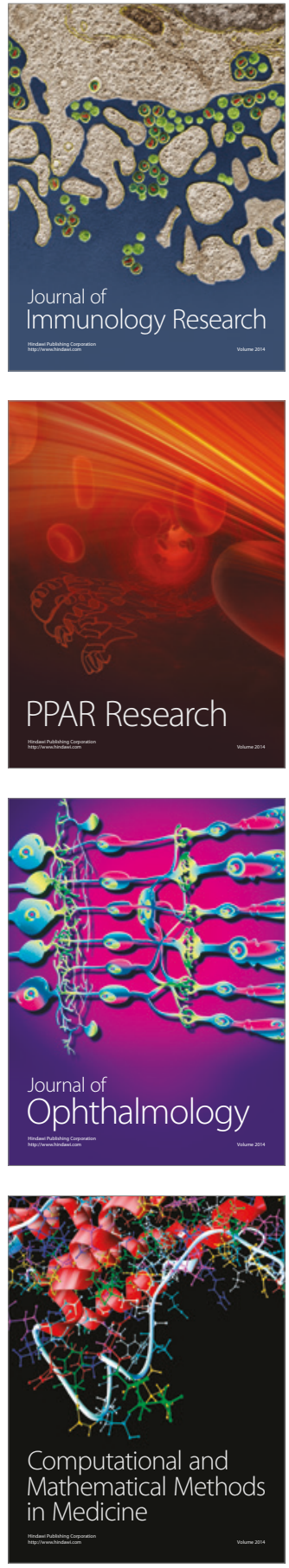

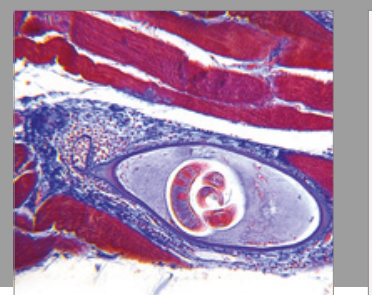

Gastroenterology Research and Practice
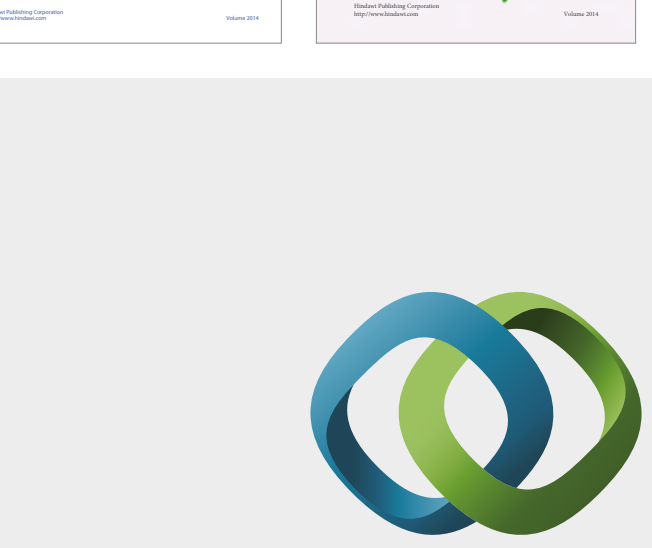

\section{Hindawi}

Submit your manuscripts at

https://www.hindawi.com
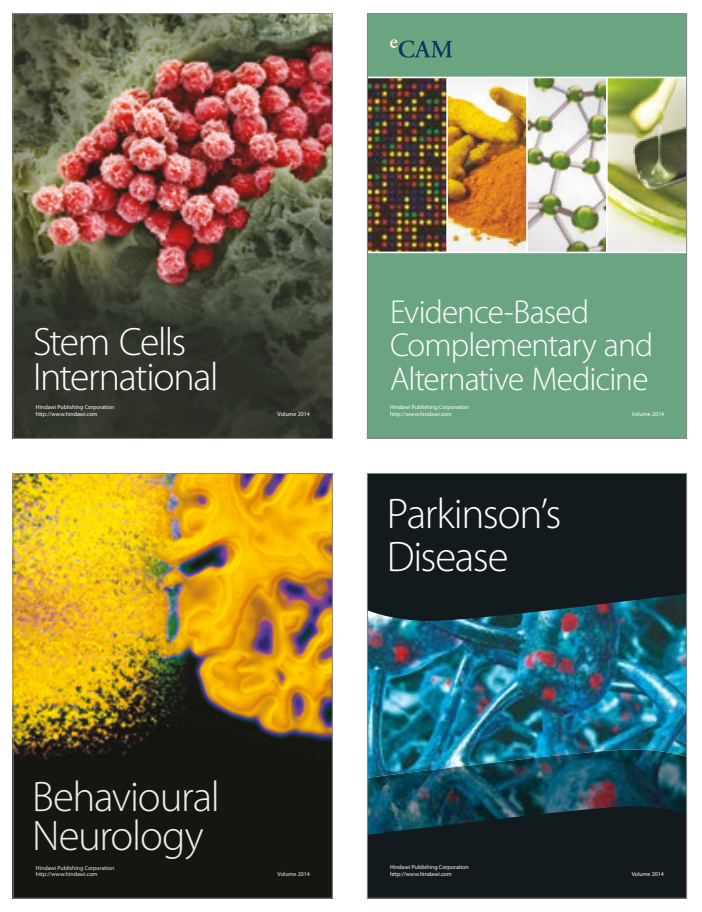
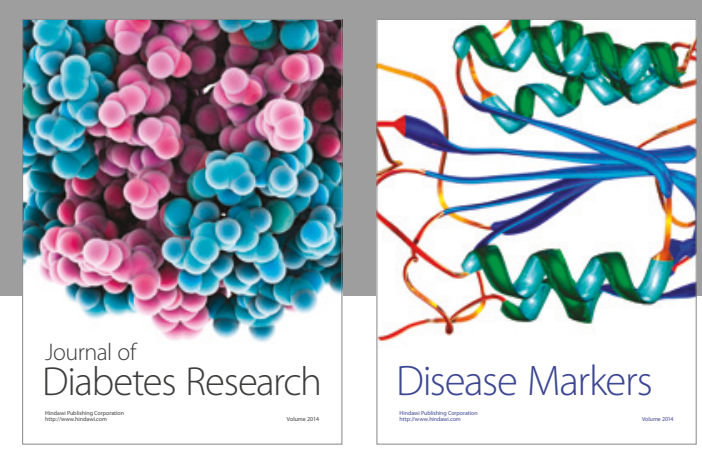

Disease Markers
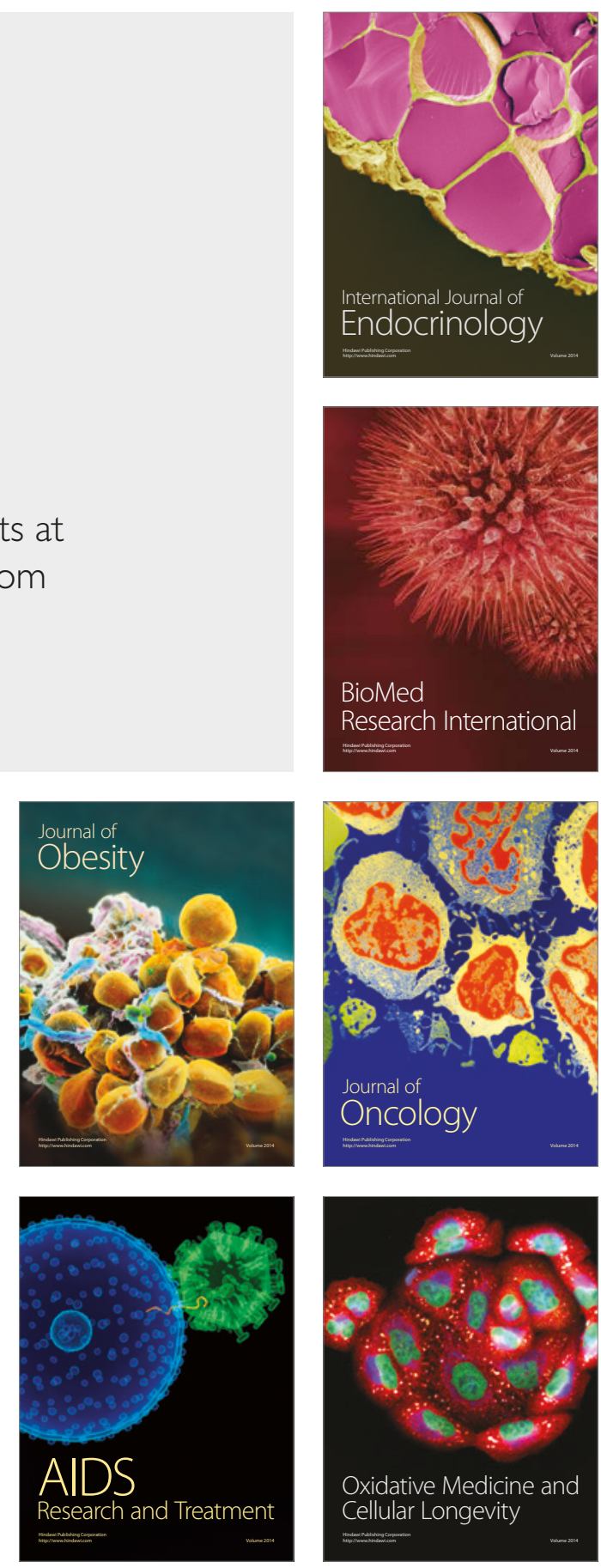